\title{
REVIEW
}

\section{PEDIATRIC CARDIAC POSTOPERATIVE CARE}

\author{
José Otávio Costa Auler Jr., Alessandra Costa Barreto, Solange Coppola \\ Gimenez and Deipara Monteiro Abellan
}

RHCFAP/308

AULER Jr. JOC et al. - Pediatric cardiac postoperative care. Rev. Hosp. Clín. Fac. Med. S. Paulo 57(3): 2002.

The Heart Institute of the University of São Paulo, Medical School is a referral center for the treatment of congenital heart diseases of neonates and infants. In the recent years, the excellent surgical results obtained in our institution may be in part due to modern anesthetic care and to postoperative care based on well-structured protocols.

The purpose of this article is to review unique aspects of neonate cardiovascular physiology, the impact of extracorporeal circulation on postoperative evolution, and the prescription for pharmacological support of acute cardiac dysfunction based on our cardiac unit protocols. The main causes of low cardiac output after surgical correction of heart congenital disease are reviewed, and methods of treatment and support are proposed as derived from the relevant literature and our protocols.

DESCRIPTORS: Pediatrics patients. Heart congenital disease. Cardiac surgery. Postoperative care.

The Heart Institute of University of São Paulo Medical School is a referral center for the treatment of neonate and pediatric congenital cardiopathies for the state of São Paulo, as well as for other regions of Brazil that do not have access to specialized centers in this type of care. Over the past 5 years, 2298 children have undergone cardiac surgery here, including 1592 who underwent surgeries with cardiopulmonary bypass (CPB) and 706 without $\mathrm{CPB}$. In the last 12 months, for example, the following surgeries were performed: correction of an atrial septal defect (ASD) (52), correction of a ventricular septal defect (VSD) (59), correction of tetralogy of Fallot (94), transposition of the great arteries (TGA) (23), and correction of atrioventricular canal defects (AV canal) (38).

Over the years, several protocols especially concerning human resources and intensive care have been adopted in our ICU that have improved postoperative results. Progress depends on harmony among several factors such as accurate diagnosis, efficient planning and scheduling of surgery, and postoperative support. The challenge for the multi-professional team is to achieve the child's full reintegration into the family with preserved neurological, affective, psychological, and social capacity. The purpose of this study was to review some unique aspects of neonatal cardiovascular physiology, since some of the children with heart disease are neonates, as well as to review specific aspects of postoperative cardiac care of neonates.

From the Anesthesia and Surgical Intensive Care Unit, Heart Institute (INCOR), Hospital das Clínicas, Faculty of Medicine, University of São Paulo.

\section{Neonatal heart}

The neonatal heart presents physiological characteristics (Table 1) that differ significantly from children of other ages or from adults, and these should be considered in the peri-operative approach $^{1}$.

First, the contractile force of the neonatal heart is less than that of the adult because of a series of structural alterations. The neonatal heart has 50\% fewer myofibers and a greater quantity of nonconnective tissue, which limits the systolic capacity, for example in obstructive cardiopathies. The myofibers are arranged in a chaotic or nonlinear pattern. Additionally, the neonatal myocardium has a smaller number of sarcomeres and mitochondria, and the calcium storage capacity of the sarcoplasmatic reticulum is immature. The immature sympathetic nervous system reduces the myocardial 
Table 1 - Neonatal heart physiological considerations.

- Decreased myocardial contractile force.

- $50 \%$ reduction in myofibers and a greater quantity of nonconnective tissue.

- Myofibers present in a chaotic or nonlinear arrangement.

- Small number of sarcomeres, and mitochondria being immature for calcium storage.

- Immature sympathetic nervous system with reduced catecholamine storage.

- Cardiac output dependent on elevated heart rate and catecholamines rather than preload.

- Parasympathetic enervation developed.

- Reduced diastolic reserve due to low ventricle compliance.

- Straight ventricular interdependency: overload in volume or pressure imposed on one of the ventricles influences filling characteristics of the other ventricle.

- Myocardium utilizes carbohydrates and amino acids for contraction.

- Elevated stores of glycogen and reduced number of mitochondria suggestive of adaptation to anaerobic conditions.

storage of catecholamines. Moreover, myocardial contractility during the first week of life occurs because of circulating catecholamines, especially epinephrine, and adequate cardiac output is maintained primarily by an elevated heart rate. Newborns are more dependent on the action of circulating catecholamines for determining heart rate than on preload for maintenance of adequate cardiac output. In contrast, parasympathetic enervation is similar to that of the adult, making the neonate more susceptible to parasympathetic stimuli.

Second, the myocardial fibers have greater length at baseline conditions, resulting in less diastolic reserve for volume overload. Therefore, contractility and ventricular compliance are effectively reduced, so the neonate myocardium is near its functional limit at baseline conditions.

Third, neonatal and fetal myocardial function is characterized by ventricular interdependency ${ }^{2}$. An overload in volume or pressure imposed on one of the ventricles influences filling characteristics of the other ventricle. In this way, the dilated right ventricle increases the filling pressure of the left one, and this increased pressure then generates elevated pressure on the right side in order to maintain transatrial flow.

Fourth, immature fetal and neonatal myocardium uses the metabolites of carbohydrates and amino acids (glycogen, glutamate, pyruvate, and lactate) for contraction. Elevated stores of glycogen and reduced numbers of mitochondria reflect adaptation to anaero- bic conditions, with greater recovery capacity and tolerance for hypoxic and ischemic insults. Therefore, the neonate is more vulnerable to hypoglycemia and reacts to stress situations with rapid alterations in $\mathrm{pH}$, lactic acid, glycemia, and temperature. These characteristics are perpetuated beyond the neonatal period. Gradual transformations occur during all the first year of life, and complete maturity of the myocardium occurs only after 2 years of age $e^{3,4}$.

\section{Cardiopulmonary Bypass}

The development of cardiopulmonary bypass (CPB) has been essential for the treatment of the great majority of congenital heart diseases. Management of CPB for congenital heart disease differs from that for adults because of the problems of aortopulmonary shunts, the immature cardiovascular system, and the eventual use of deep hypothermic circulatory arrest. During deep hypothermic circulatory arrest, the blood available in the systemic veins (generally the cavas vein) is drained to the oxygenator, which provides oxygen, removes carbon dioxide, and reduces the blood temperature. In the process, the oxygenated blood returns to the aorta by a system of pumps that generates continuous flow from the CPB to the patient.

The plastic polyvinyl circuits of the CPB system are full of a balanced solution made of colloids and crystalloids, the composition of which varies according to the age and weight of the pa- tient, maintaining a $\mathrm{pH}$ and electrolyte composition similar to that of the plasma.

To reduce the metabolic rate and for protection of tissues during $\mathrm{CPB}$, moderate $\left(25^{\circ} \mathrm{C}\right.$ to $\left.32^{\circ} \mathrm{C}\right)$ or profound $\left(15^{\circ} \mathrm{C}\right.$ to $20^{\circ} \mathrm{C}$ ) hypothermia is used. Profound hypothermia permits reduction of flow through the $\mathrm{CPB}$, reduces trauma to blood elements, and allows total circulatory arrest, usually during aortic arch repair or in very small children. Hypothermia also preserves the highly energetic phosphate stores and reduces liberation of toxic cerebral neurotransmitters.

Different methods (eg, pH-stat, alphastat) have been evaluated in the past several decades for correction of acidbase equilibrium during hypothermia, primarily by comparing the neurological evolution of patients. According to $\mathrm{du}$ Plessis et $\mathrm{al}^{5}$, children undergoing surgery using the alpha-stat method have poorer cognitive development in the late postoperative follow-up period compared to those undergoing the $\mathrm{pH}$ stat method, probably because of the acidification that the $\mathrm{pH}$-stat method provides, favoring vasodilatation and homogeneous hypothermic cerebral protection $^{5}$.

In general, CPB alters all physiological processes of the organism and may lead to organic dysfunction of different magnitudes. The morbidity associated with CPB is largely associated with damage to blood elements and proteins caused by blood gas alterations and the prosthetic surface interface.

The exposure of blood to various abnormal conditions is implicated in a systemic inflammatory response. This response includes interstitial edema, coagulation cascade activation that generates kallikrein and bradykinin, and platelet consumption. Neutrophils and monocytes are also activated to express reactive cellular receptors and release vasoactive and cytotoxic substances. Activated complement proteins and products from neutrophils and monocytes mediate much of the inflammatory response to $\mathrm{CPB}$. Hemodilution used during $\mathrm{CPB}$, on the other hand, reduces microcirculation resistance 
Table 2 - Excessive hemodilution during and after cardiopulmonary bypass may cause:

- More requirement for blood transfusion.

- Greater use of inotropic agents.

- Undesirable hemodynamic performance.

- Depressed myocardial contractility.

while exacerbating interstitial edema. The formation of edema can be explained by the hemodilution (Table 2) and by the inflammatory process unleashed by the passage of blood and elements through the nonendothelial surfaces of the CPB circuits. Permeability of the capillary vessels is greater in immature individuals than in adults. Potent vasoactive substances are activated, including the anaphylatoxins $\mathrm{C} 3 \mathrm{a}$ and $\mathrm{C} 5 \mathrm{a}$, tumor necrosis factor, and free radicals derived from oxygen, and neutrophils are activated (Table 3 ). Conventional ultrafiltration has been used in an attempt to prevent this edema; however, its effectiveness seems limited. In 1993, Naik and Elliot ${ }^{6,7}$ modified the ultrafiltration system, placing the hemoconcentrator between the aorta and the right atrium immediately following CPB (Table 4). With this modification, the following have been noted: a reduction in total body water content, improved systolic function, increased diastolic compliance, increased systemic arterial pressure, hematocrit, fibrinogen, and total plasmatic protein, and decreased levels of endothelial serum cytokines and the $\mathrm{C} 3 \mathrm{a}$ and $\mathrm{C} 5 \mathrm{a}$ fractions of complement ${ }^{6,7}$.

The more prolonged the CPB procedure is, the greater are the deleterious effects on these factors, since CPB imposes an overload on the systemic circulation that is often already compromised by the previous heart failure.

\section{Immediate postoperative care and monitoring}

Adequate monitoring during the postoperative period involves a combination of clinical or auxiliary methods for evaluating the surgical correction, myocardial function, and the relationship between systemic and pulmonary blood flow ${ }^{8}$. Standard monitoring in the postoperative period is similar to that during anesthesia and surgery. Sometimes, depending on the clinical evolution, more sophisticated monitoring may be added to facilitate clinical diagnosis and treatment. Standard monitoring consists of ECG, direct arterial pressure, temperature probe, and central venous pressure. Pulse oximeters and sometimes capnography are useful for accompanying mechanical ventilation. To obtain direct arterial pressure, radial catheters are generally preferable. Peripheral arterial catheters may not precisely reflect aortic pressure, particularly when placed in infants' feet and are even less accurate in the presence of hypothermia. Temperature monitoring is very important for assessing the metabolism. Probes can be placed in the rectum or near the esophagus to provide adequate assessment of body and core temperature. A nasopharyngeal probe gives an estimate of brain temperature. The central venous line is generally established by insertion of a catheter utilizing the Seldinger technique into the internal jugular vein of choice. Depending on the degree of severity and type of cardiac disease, transthoracic or transvenous measurement of pulmonary and left atrial pressures may give useful physiologic information. Cardiac output can be also be determined by thermodilution. In small children, the traditional Swan-Ganz 7 or $5 \mathrm{~F}$ catheter cannot be used, but cardiac output can be measured by means of a thermodilution $2 \mathrm{~F}$ probe inserted by the surgeon into the pulmonary artery. Newer techniques including echocardiography and on-line monitoring of arterial and mixed venous saturation obtained by fiberoptic catheters are increasingly used in postoperative care.

In postoperative care of the pediatric patient, clinical evaluation must be complete and systematic. Consequently, complications can be foreseen, and catastrophic situations can be avoided. Care should be initiated while the child is still in the operating room, with special attention to rewarming to $36.5^{\circ} \mathrm{C}$, control of bleeding, ventilation, and acid-base and electrolyte balance. It is very important during this phase to stabilize cardiac function through maintaining correct intravascular volumes, adequate heart rate, and adequacy of myocardial contractility.

The information concerning the anesthetic and surgical procedure that should be transmitted in detail to the multi-professional ICU team includes surgical technique, type of anesthesia, perfusion and aortic clamping time, water and colloid balance, diuresis, venous and arterial catheter placement, pacemaker wire and mediastinal or thoracic drainage tube positioning, ventilatory

Table 3 - Causes of excessive edema in neonates and small children under cardiopulmonary bypass:

- Permeability of the capillary vessels is greater in immature individuals than for adults.

- Potent vasoactive substances are activated during CPB including anaphylatoxins, C3a and C5a, TNF, and other cytokines that contribute to capillary leaking.

Table 4 - Modified ultrafiltration in pediatric open heart operation results in:

- Reduction in total body water content.

- Improvement in systolic function.

- Increase in diastolic compliance.

- Increase in systemic arterial pressure.

- Elevation in hematocrit, fibrinogen, and total plasmatic protein concentration.

- Decrease in the cytokines C3a and C5a fraction of complement. 
conditions, acid-base balance, heart rate and arrhythmias, and coagulation and vasoactive medication in use $\mathrm{e}^{9}$.

Supplementary monitoring, including left atrial and pulmonary arterial pressure and serial echocardiography, is indicated in all children undergoing more serious or complex cardiopathy correction that present: a) significant myocardial dysfunction (left coronary anomaly, transposition of the great arteries, hypoplastic left heart); b) pulmonary arterial hypertension during or prior to the operative period (total anomalous drainage of the pulmonary veins, aortic arch interruption, truncus arteriosus communis); c) valvoplasty or correction of an atrioventricular canal defect with a residual defect of mitral insufficiency.

\section{Cardiovascular function}

The cardiovascular function of a child can be considered adequate in the postoperative period when cardiac output is sufficient to supply the demands for cellular oxygen.

The main determining factors of cardiac output are preload, afterload, contractility, heart rate, and diastolic function $^{1}$. Other indirect factors, such as anxiety, pain, temperature, hemoglobin level, endogenous, and exogenous catecholamines, and blood biochemical composition, can alter the myocardial oxygen supply-demand relationship in addition to affecting the cardiac output $^{10}$.

During the postoperative period, cardiovascular dysfunction represented by low cardiac output can develop for a series of reasons. First are all of the pre-operative conditions, including the structural defect itself and the functional cardiac state, the general birthing conditions, transfer from hospital to specialized care centers, the detail and efficiency of planning for the surgery, and associated complications such as respiratory insufficiency, infection, and metabolic and electrolyte disturbances. Secondly, the dysfunction is dependent on the surgery performed, degree of ventricle muscle incision, placement of corrective patches, myocardial protection, CPB time and aortic clamping, level of hypothermia during $\mathrm{CPB}$, use of total circulatory arrest, type of anesthesia, and intra-operative complications.

\section{Cardiovascular Evaluation}

Postoperative cardiovascular function can be evaluated by clinical examination, related tissue oxygen indexes, echocardiography and hemodynamic and/or radioisotope evaluation $^{10}$.

Important clinical signals for the evaluation of cardiac output are perspiration, adequate level of consciousness, coloring and temperature of extremities, thermal gradient between knees and feet, central and peripheral thermal gradient, amplitude of peripheral pulse, capillary filling, arterial pressure, and urinary output. Accordingly, cardiac output is considered adequate when there is no cold perspiration or psychomotor agitation, the members of extremities are warm and colored, the feet are hotter than the knees, the central-peripheral thermal gradient is less than $4^{\circ} \mathrm{C}$, the peripheral pulse is easily palpable, capillary filling is satisfactory, arterial pressure is within the normal limits for the age group, and urinary output is greater than $1 \mathrm{~mL} / \mathrm{kg} /$ hour. It is important to remember that adequate peripheral vasodilatation only occurs after the fourth postoperative hour, with normal re-establishment of tissue perfusion around the sixth postoperative hour ${ }^{10}$.

Among the related tissue oxygen indexes, lactate is a marker of anaerobic metabolism and tissue energy deficit. It reflects hypoxia, ischemia, or aggressive action of a toxic tissue agent. Values for serum lactate above $2.0-3.0 \mathrm{mmol} / \mathrm{L}$ reflect significant evidence of tissue hypoxia. Following total circulatory arrest, profound hypothermia $\left(<20^{\circ} \mathrm{C}\right)$, or low cardiac output, serum lactate levels commonly exceed 6-10 $\mathrm{mmol} / \mathrm{L}$. Also regarding tissue oxygenation, the calculation of the arterial-venous oxygen differential, as well as the consumption, transport, and peripheral extraction of oxygen can be used in evaluation of low cardiac output in small children. Clinical outcome can be related to the relationship of these indexes to normal levels.

Measurement of troponin could be a specific marker for the evaluation of myocardial injury, according Taggart et $\mathrm{al}^{11}$. They found that infants undergoing cardiac surgery with CPB had elevated levels of troponin during the postoperative period that remained elevated for 72 hours. Infants undergoing cardiac surgery without $\mathrm{CPB}$, did not have elevated levels of troponin. On the other hand, infants from both groups had elevated creatine kinase MB isoenzyme levels that progressively decreased over 72 hours.

Two-dimensional and Doppler echocardiography are valuable tools for postoperative cardiac functional and structural evaluation. These methods permit analysis of cardiac chambers and operative results, detection of residual defects, evaluation of position and function of valvar prosthesis, segmental and global myocardial analysis, calculation of shortening and the ventricular ejection fraction, and estimation of pressures inside cardiac chambers.

Cardiac catheterization for angiography or manometry of cardiac cavities is a traditional and accepted method that may be used in the postoperative period. The principal indications for cardiac catheterization are evaluation of systemic-pulmonary shunt permeability, for example in the Blalock-Taussig operation, evaluation of pulmonary vascularization following reunification or centralization of pulmonary arteries, measurement of residual pressure gradients after amplification of the right or left atrium, and evaluation of cardiopulmonary anastomosis, for example in the Fontan procedure or modified Glenn operation. During the neonatal period, new types of cardiac hemodynamic procedures are occasionally indicated for therapy or for investigations into residual defects that cannot be clarified 
using other methods of diagnosis.

Direct measurement of cardiac output can be obtained by invasive and noninvasive methods. The noninvasive method uses a relationship between expected oxygen consumption and the arterial-venous oxygen differential. However, the method may be incorrect when used to determine the expected consumption in the postoperative period. Noninvasive methods for determining oxygen consumption calculated by nomograms are dependent on heart rate, age, sex, and body temperature; some of these factors are quite variable in the first hours following $\mathrm{CPB}^{12}$.

As previously mentioned, the thermodilution method for determining cardiac output is most frequently used in postoperative care; in pediatrics, the data are indexed to body surface area. Accordingly, the cardiac index is considered normal in children when it is above $3 \mathrm{~L} / \mathrm{min} / \mathrm{m}^{2}$, moderately reduced between 2.0 to $3.0 \mathrm{~L} / \mathrm{min} / \mathrm{m}^{2}$, and severely reduced when lower than $2.0 \mathrm{~L} /$ $\mathrm{min} / \mathrm{m}^{2}$. In general, the cardiac index tends to be lower in the fourth postoperative hour in relation to the immediate postoperative period and increases after the 9th or 12th hours. Kirklin et $\mathrm{al}^{12}$ correlated a greater number of hospital deaths with a cardiac index below $2.0 \mathrm{~L} / \mathrm{min} / \mathrm{m}^{2}$ in a postoperative study of 174 children under the age of 3 months.

\section{Low cardiac output}

Low postoperative cardiac output is primarily caused by reduction in myocardial contractility caused by one of the mechanisms or factors above mentioned. Severe myocardial dysfunction can be observed, for example, in more complex congenital heart disease that demands a lengthy CPB procedure and aortic clamping. Left anomalous coronary artery in the pulmonary trunk, hypoplastic left heart syndrome, transposition of the great arteries, severe tetralogy of Fallot, and severe pulmonary hypertension are all associated with significant risk of poor cardiac function after surgery.
Secondarily, hemodynamic instability is caused by inadequate intravascular volume due to a series of factors including an endothelial inflammatory process resulting from the CPB procedure, which transfers fluids to the interstitial area (primarily in the first 24 hours); vasodilatation during the rewarming period after CPB (4 to 6 hours postoperatively); urinary output; and active blood loss.

Control of intravascular volume and indirectly of preload should promote more adequate systolic volume, according to the Frank-Starling law. In the absence of more severe valvar injury, the final diastolic pressure of the ventricles corresponds to the mean pressure of the atrium; therefore, volume can be controlled via right and left atrial pressures. During the postoperative period, the atrial pressure should remain around $15 \mathrm{mmHg}$, although it can reach $18 \mathrm{mmHg}$ in the right atrium and 20 $\mathrm{mmHg}$ in the left when there is hypertrophy or hypocontractility, partial obstruction in the ventricular outflow, or pulmonary artery hypertension.

Afterload is a third factor influencing cardiac output. It can be elevated by vasoconstriction secondary to $\mathrm{CPB}$, hypothermia, excessive endogenous catecholamines, or administration of vasoactive amines. The heart with any degree of myocardial dysfunction can experience significant reduction of myocardial fiber length and consequently its systolic volume when subjected to any increase in afterload.

Heart rate is dependent on factors such as use of digital or beta-blocking agents in the preoperative period, type of surgery, perioperative rhythm disturbances, volume, temperature, pain, anxiety, anemia, metabolic disturbances, and use of vasoactive agents with chronotropic action.

Alternatively, postoperative myocardial edema could be responsible for ventricular diastolic restriction.

\section{Treatment}

Therapeutic measures for low postoperative cardiac output include 3 con- comitant and related procedures: 1) diagnosis, 2) reduction in metabolic demand, 3) adequate tissue perfusion and oxygen transport ${ }^{1}$.

Suspected cardiac dysfunction should be promptly investigated for etiological diagnosis by clinical or supplemental methods so specific and effective conduct can be adopted.

Reduction in metabolic demand requires the use of measures that favor adequate temperature and reduction in respiratory workload. Initial measures, even during diagnostic investigation can be adopted to maintain body temperature around $36.5^{\circ} \mathrm{C}^{10}$.

Ventilation and respiratory mechanics play a primary role in the improving the hemodynamic state of the postoperative patient through use of positive end expiratory pressure and adjustment of $\mathrm{PaCO}_{2}$ and $\mathrm{pH}$. Factors that influence the hemodynamic state include general anesthesia, pulmonary retraction, edema due to residual injury, reduced functional residual capacity leading to alterations in gas exchange, pulmonary compliance, abnormalities in the ventilation-perfusion ratio, and intrapulmonary shunts ${ }^{10}$.

Mechanical ventilation is maintained until bleeding is controlled, hemodynamics are stabilized, adequate body temperature attained, metabolic disturbances are corrected, and acidbase balance is achieved.

The necessary support for adequate tissue perfusion and oxygen transport is basically related to concomitant employment of inotropic and vasodilator agents (Table 5). Inotropic agents are the primary therapeutic tools for treating low cardiac output. The principal sympathomimetic amines are represented by dopamine and epinephrine or synthetic agents such as isoproterenol and dobutamine. All of these agents act through stimulation of myocardial beta-adrenergic receptors, increasing cyclic adenylate and cyclic adenosine monophosphate (cAMP) ${ }^{13}$.

Dopamine increases myocardial contractility through several mechanisms including stimulation of postsynaptic beta 1 -adrenergic receptors, in- 
Table 5 - Agents utilized in pharmacological manipulations for increasing right ventricular inotropism, and/or controlling pulmonary vascular resistance:

- $\quad$ Sodium nitroprusside.

- Nitroglycerin.

- Phosphodiesterase inhibitors.

- Prostaglandin E1 (PGE1).

- Prostacyclin (PGI2).

- Ultra-short-acting intravenous vasodilators: adenosine and adenosine triphosphate

- Inhaled nitric oxide.

Table 6 - Phosphodiesterase inhibitors-cardiovascular effects:

- Increase myocardial contractility.

- Increased lusitropic activity (energy-dependent diastolic relaxation).

- Vasodilating properties.

- Reduce ventricular wall stress.

- Reduce filling pressures.

- Reduce impedance to ventricular ejection

creasing liberation of norepinephrine in the myocardial presynaptic sympathetic storage sites and decreasing enzymatic degradation and reabsorption of norepinephrine. Dopamine also acts as a pulmonary and systemic vasoconstrictor through action in alpha 1- and alpha 2-postsynaptic receptors. Because of its action on dopamine adrenergic receptors, dopamine acts as a renal, spleen, coronary, and cerebral vasodilator. In animal research, dopamine has been shown to be less efficient in neonates than in adult animals, probably because of the greater clearance, reduced myocardial adrenergic enervation, lower density of myocardial beta 1 -adrenergic receptors, and differing maturation among peripheral alpha receptors and myocardial beta 1 and dopaminergic receptors. In neonates, dopamine increases cardiac output, heart rate, and systemic arterial pressure ${ }^{10}$. Dopamine is indicated in moderately low cardiac output, especially when there is water retention from the cardiac insufficiency itself or because of the CPB procedure. In doses of 1 to $2 \mu \mathrm{g} / \mathrm{kg} / \mathrm{min}$, dopamine acts as vasodilator on the mesenteric and renal circulation. In doses between 2 and $10 \mu \mathrm{g} /$ $\mathrm{kg} / \mathrm{min}$, it acts on myocardial beta 1 receptors producing increased contractility and coronary flow with consequent improvement in mean arterial pressure and cardiac index. However, Driscoll et al. ${ }^{14}$, observed that the neo- natal myocardium seems less sensitive to the action of dopamine than does the myocardium of older children, and more elevated doses are necessary to obtain the same effects. In another study, Lang et al. ${ }^{15}$ observed that dopamine increases heart rate, cardiac index, and systemic arterial pressure after pediatric cardiac surgery when given in doses greater than $15 \mu \mathrm{g} / \mathrm{kg} /$ min but does not have significant action on systemic vascular resistance. In higher doses, dopamine raises pulmonary resistance, which is why it is not used in cases of previous pulmonary artery hypertension.

Dobutamine is a synthetic sympathomimetic amine, beta 1 -adrenergic agonist. It differs from dopamine by not liberating endogenous norepinephrine and has little peripheral action. Dobutamine increases cardiac output and reduces systemic vascular resistance and ventricular filling pressure. Since it exerts a positive chronotropic effect, elevating heart rate, its use could be limited in the postoperative cardiac surgery period in children and neonates ${ }^{12}$. Dobutamine is indicated for low cardiac output when not accompanied by severe hypotension. In sepsis without cardiac failure, dobutamine is the therapy of choice, and when cardiac compromise already exists, it can be indicated when associated with a vasoconstrictor like norepinephrine. Increase in cardiac output and reduction in systemic vascular resistance can be obtained by starting dobutamine at low doses such as $2.5 \mu \mathrm{g} / \mathrm{kg} / \mathrm{min}$. In general, doses of 2 to $20 \mu \mathrm{g} / \mathrm{kg} / \mathrm{min}$ are effective.

Isoproterenol is a synthetic analogue of norepinephrine. It is an agonist that stimulates myocardial beta 1adrenergic receptors, with direct inotropic and chronotropic action, and promotes peripheral vasodilatation mediated by beta 2 -adrenergic receptors. It increases cardiac output and systolic arterial pressure, and it vasodilates renal, spleen, and skeletal muscle tissue with a reduction in diastolic arterial pressure and systemic vascular resistance ${ }^{13}$. Isoproterenol is indicated mainly in the presence of sinusal bradycardia or transitory atrioventricular block. In the neonatal population, it can be indicated for controlling low cardiac output secondary to persistent arterial hypertension in the newborn. The initial dose of isoproterenol is 0.01 to 0.05 $\mu \mathrm{g} / \mathrm{kg} / \mathrm{min}$ for symptomatic bradycardia and 0.05 to $0.1 \mu \mathrm{g} / \mathrm{kg} / \mathrm{min}$ as a positive inotropic agent. With the increase in heart rate, an accentuated increase in myocardial oxygen consumption and transitory myocardial ischemia may occur. Sinus tachycardia ventricular arrhythmias due to compromised coronary flow can appear with more elevated doses.

Epinephrine is an endogenous catecholamine that is liberated from the adrenal medulla and derived from norepinephrine. It acts on alpha-, beta 1- and beta 2 -adrenergic receptors. Depending on the dose, it increases heart rate and systolic arterial pressure, decreases diastolic arterial pressure, and relaxes the peripheral vascular bed. At high doses the alpha-adrenergic effect predominates, producing skin, gastrointestinal, and renal perfusion ${ }^{10}$. Epinephrine is indicated for low cardiac output accompanied by severe systemic arterial hypotension that compromises coronary perfusion, especially in postoperative cardiac surgery with cardiogenic or septic shock that is not responsive to dopamine and dobutamine. It is also effective in cardiac arrest. At doses of 
0.03 to $0.1 \mu \mathrm{g} / \mathrm{kg} / \mathrm{min}$, beta 1 and beta 2 effects are predominant. At doses of 0.1 to $0.2 \mu \mathrm{g} / \mathrm{kg} / \mathrm{min}$, there is a mixed alpha and beta effect. At doses above 0.2 up to $1 \mu \mathrm{g} / \mathrm{kg} / \mathrm{min}$, the alpha response is predominant. Ventricular arrhythmia possibly caused by subendocardic ischemia and peripheral vasoconstriction may be observed with this agent.

Norepinephrine it is a local adrenergic neurotransmitter. It has beta 1 and alpha action, which increases the systolic arterial pressure as much as the diastolic pressure. Cardiac output can increase or decrease depending on the myocardial reserve. A starting dose of 0.05 to $0.1 \mu \mathrm{g} / \mathrm{kg} / \mathrm{min}$ has been recommended.

Another possibility for therapy in the control of low cardiac output is the use of phosphodiesterase inhibitors, introduced into clinical practice in 1984. Phosphodiesterase agents, such as amrinone and milrinone inhibitors, are non-glycoside non-sympathomimetic agents that selectively inhibit the cyclic phosphodiesterase nucleotide, increasing myocardial and vascular cAMP, independent of the beta receptors. Elevation of cAMP increases contraction through calcium regulation by 2 mechanisms: first, by activation of the kinase protein that facilitates the rapid entry of calcium through calcium channels, and second, by activation of the calcium stores of the sarcoplasmic reticulum. Accordingly, the phosphodiesterase inhibitors have 3 actions: increasing inotropism and contractility, increasing arteriolar and venous vasodilatation, and increasing ventricular relaxation during diastole (Table 6). Amrinone increases the cardiac index and decreases left ventricular end diastolic pressure, pulmonary capillary pressure, and right atrial pressure in congestive heart failure and in postoperative low cardiac output. Additionally, when associated with dobutamine, it has greater action on the elevation of the cardiac index and on the reduction in systemic vascular resistance, and it does not significantly increase heart rate $^{16}$.
Milrinone is considered to be 10 to 30 times more potent than amrinone. It does not elevate myocardial consumption and is a coronary vasodilator. Milrinone is metabolized primarily in the kidneys, necessitating dosage correction in the presence of renal failure. Amrinone is metabolized by the liver and excreted primarily by the kidneys.

Phosphodiesterase inhibitors can be indicated for low cardiac output with myocardial dysfunction and elevated systemic vascular resistance, but without severe arterial hypotension. Pediatric dosages of amrinone are variable in the literature but have been cited as: $0.75 \mu \mathrm{g} / \mathrm{kg}$ bolus dose (over time for 2 3 doses) followed by infusion of 5-10 $\mu \mathrm{g} / \mathrm{kg} / \mathrm{min}$ for maintenance. In neonates, bolus doses of 3 to $4.5 \mu \mathrm{g} / \mathrm{kg}$ are used, followed by 5 to $15 \mu \mathrm{g} / \mathrm{kg} / \mathrm{min}$ of continuous infusion. Since the half-life of this agent is relatively long ( 3 to 15 hours), special attention must be given to those children that already have systemic arterial hypotension. In adults with normal renal function, the bolus of milrinone is $50 \mu \mathrm{g} / \mathrm{kg}$ over $10 \mathrm{minu}-$ tes, followed by 0.375 to $0.75 \mu \mathrm{g} / \mathrm{kg} / \mathrm{min}$ infusion. Side effects can include hypotension caused by vasodilatation, necessitating administration of fluids; thrombocytopenia, usually 7 to 10 days after its administration; and elevation of hepatic enzymes, as described for amrinone infusion (Table 7).

Vasodilators for low cardiac output are adjuvants to inotropic therapy, reducing systemic arterial resistance and enhancing ventricular ejection. Sodium nitroprusside in doses of 0.5 to $8 \mu \mathrm{g} / \mathrm{kg} / \mathrm{min}$ acts to increase vascular relaxation through cGMP, causing arteriolar and venous vasodilatation.
The start of action after endovenous administration occurs in a few minutes. The primary metabolites of nitroprusside are thiocyanate and cyanide, which have been detected during prolonged infusion ${ }^{13}$.

Sodium nitroprusside is the vasodilator most used, not only for controlling systemic and pulmonary hypertensive states, but also for reducing afterload in low cardiac output, primarily after cardiac surgery. Nowadays for pediatric postoperative cardiac patients, sodium nitroprusside has progressively been replaced by phosphodiesterase inhibitors, which are not only inotropic but also are arteriolar and venous vasodilators.

Nitroglycerine in doses of 0.5 to $20 \mu \mathrm{g} / \mathrm{kg} / \mathrm{min}$ is a vasodilator that acts by releasing nitric oxide. The main hemodynamic effect is vasodilation with reduction in ventricular filling pressures. Nitroglycerine is indicated for situations of increased preload and signs of pulmonary and systemic venous congestion.

\section{The utilization of inhaled nitric oxide in postoperative period}

Nitric oxide (NO) produced by the endothelial cells exerts important functions on cardiovascular system. In lung tissue, the maintenance of blood vessels in a relaxed status is fundamentally important, especially in the presence of previous pulmonary hypertension and/ or right ventricle dysfunction. Right ventricular (RV) dysfunction is frequently observed in infants and children in the postoperative period. Pharmacological and ventilatory manipulations are directed at increasing $\mathrm{RV}$ inotropism, uti-

Table 7 - Pharmacology of amrinone and milrinone.

\begin{tabular}{lcc}
\hline \multicolumn{1}{c}{ Variable measured } & Amrinone (IV) & Milrinone(IV) \\
\hline & & \\
Loading dose, mg/kg (per approved product labeling) & $1.5(0.72 \times 2)$ & 0.050 \\
Loading dose (maximally effective) & 3.0 & 0.075 \\
Infusion rate, mg/kg/min (labeling) & $5-10$ & $0.375-0.75$ \\
Incompatible with dextrose solutions & Yes & No \\
Average half-life in CHF patients (h) & $5-8$ & $2-3$ \\
Incidence of thrombocytopenia & $2.6 \%$ & $0.4 \%$ \\
Incidence of ventricular tachycardia/fibrillation & $0.8 \%$ & $3.6 \%$ \\
\hline
\end{tabular}


Table 8 - Inhaled nitric oxide in post-op after cardiac surgery: Main indication Pulmonary hypertension accompanied of right ventricular dysfunction

- Correct metabolic disturbances.

- Provide sedation and adequate ventilation.

- Consider using phosphodiesterase inhibitors first.

- Start inhaled NO with low doses.

- Closely monitor with echocardiography.

- Remove NO carefully.

lizing inotropic drugs as outlined above, by optimizing RV preload, and by controlling pulmonary vascular resistance (PVR). In the presence of RV dysfunction, control of PVR is extremely important, since RV output is very sensitive to the variations of afterload. As previously discussed, the most commonly used agents for controlling PVR are sodium nitroprusside, nitroglycerin, and phosphodiesterase inhibitors ${ }^{17}$. Prostaglandin E1 (PGE1) and prostacyclin (PGI2) both have a pulmonary vasodilating ef- fect. The problem is that the effects of these drugs are not limited to the pulmonary circulation. Because of the lack of specificity of vasodilator drugs on the pulmonary bed, newer pharmacological methods of controlling elevated PVR are being recommended. Inhaled NO and ultra-short-acting intravenous vasodilators, such as adenosine and adenosine triphosphate, represent new modalities for treating acute pulmonary hypertension in postoperative pediatric cardiac patients (Table 8). Although nonselec- tive, NO is rapidly inactivated by hemoglobin; therefore, when NO is inhaled, the systemic circulation is protected from its vasodilating properties. When we administered inhaled NO to a group of patients after cardiac surgery, we observed a significant pulmonary vasodilatation and no clinically significant effects on systemic pressure ${ }^{18}$. These same effects have also been observed in children after cardiac surgery ${ }^{19}$. In more severe situations of RV dysfunction accompanied by pulmonary hypertension, we have used small doses of inhaled NO ( 3 to 5 parts per million) in our postoperative children. Although several studies have demonstrated the vasodilating properties of $\mathrm{NO}$ on pulmonary circulation, recommendation for its use as a routine agent during cardiac surgery in pediatric patients suffering pulmonary hypertension is still under investigation $^{20}$.
AULER Jr. JOC e col. - Cuidados pediátricos pós-operatórios de cirurgia cardíaca. Rev. Hosp. Clín. Fac. Med. S. Paulo 57(3): 2002.

O Instituto do Coração da Faculdade de Medicina da Universidade de São Paulo é centro de excelência para tratamento das cardiopatias congênitas que abranjam o período neonatal e pediátrico. Nos últimos anos, parte dos excelentes resultados cirúrgicos da Instituição devem-se ao progresso da anestesia e a estruturação dos cuidados pós-operatórios por meio de protocolos bem definidos.

Este artigo se propõe a revisar aspectos de interesse da fisiologia cardiovascular do neonato, as repercussões orgânicas da circulação extracorpórea, o pós-operatório imediato, incluindo avaliação cardiovascular e a te- rapia farmacológica desta Unidade. Será apresentada uma revisão das causas mais comuns de baixo débito; cirurgias para a correção das cardiopatias congênitas e propostas de métodos diagnósticos e terapêuticos, baseados na literatura especializada e protocolos da Unidade.

DESCRITORES: Pediatria. Cardiopatia congênita. Cirurgia. Pós-operatório. 


\section{References}

1. FELTES T - Postoperative recovery from congenital heart disease. In: GARSON Jr A. - The science and practice of pediatric cardiology. Baltimore, Williams \& Wilkins, 1998. p. 2387-413.

2. KLEINMAN CS - Abnormal fetal cardiovascular physiology. In: POLIN RA, FOX WW - Fetal and neonatal physiology. Philadelphia, Saunders, 1992. p. 666-70.

3. FISHER DJ, HEYMAN MA \& RUDOLPH AM - Myocardial oxygen and carbohydrate consumption in fetal lambs in adult sheep. Am J Physiol 1980; 238:H399.

4.FISHER DJ, HEYMAN MA \& RUDOLPH AM - Myocardial consumption of oxygen and carbohydrates in newborn sheep. Pediatr Res 1981; 15:843-6.

5. DU PLESSIS AJ, JONAS RA, WYPIJ D et al. - Perioperative effects of alpha-stat versus pH-stat strategies for deep hypothermic cardiopulmonary bypass in infants. J Thorac Cardiovasc Surg 1997; 114:990-1001.

6. NAIK SK \& ELLIOTT MJ - Ultrafiltration and pediatric cardiopulmonary bypass. Perfusion 1993; 8:101-12.

7.ELLIOTT MJ - Ultrafiltration and modified ultrafiltration during pediatric open-heart operations. Ann Thorac Surg 1993; 56: $1518-22$.

8.STANLEY TE \& NEWMAN MF - Monitoring of the cardiac surgery patient. In: BARASH PG, REVES JG - Cardiac anesthesia: principles and clinical practice. Philadelphia, Fawzy G.Estafanous, 1994. p.185-220.

9. REICH DL \& KAPLAN JA - Hemodynamic monitoring. In: KAPLAN JA - Cardiac Anesthesia . Philadelphia, Saunders, 1993. p.261-98.

10. WERNOVSKY G, CHANG AC \& WESSEL DL - Intensive care. In: EMMANOUILIDES GC, ALLEN HD, REIMENSCHNEIDER TA et al. - Heart disease in infants, children and adolescents. $5^{\text {th }}$ ed. Baltimore, Willians \& Wilkins, 1995. p.398-439.

11. TAGGART DP, HADJINIKOLAS L, HOOPER J et al. - Effects of age and ischemic time on biochemical evidence of myocardial injury after pediatric cardiac operation. J Thorac Cardiovasc Surg 1997; 113:728-35.
12. KIRKLIN JW \& BARRATT-BOYES BG - Postoperative care. In: KIRKLIN JW \& BARRATT-BOYES BG. - Cardiac surgery. New York, Churchill Livingstone, 1993. p.195-248.

13. TALNER NS - Heart failure. In: EMMANOUILIDES GC, ALLEN HD, REIMENSCHNEIDER TA et al. - Heart disease in infants, children and adolescents. $5^{\text {th }} \mathrm{ed}$. Baltimore, Williams \& Wilkins, 1995. p.1746-73.

14.DRISCOLL DJ, GILETTE PC \& MCNAMARA DG - The use of dopamine in children. J Pediatr 1978;92:309-14.

15. LANG P, WILLIAMS RG, NORWOOD WI et al. - The hemodynamic effects of dopamine in infants after corrective cardiac surgery. J Pediatr 1980; 96:630-34.

16. WESSEL DL, TRIEDMAN JK, WERNOVSKY G et al. - Pulmonary and systemic hemodynamics of amrinone in neonates following cardiopulmonary bypass. Circulation 1989; 80(suppl II): 488 .

17.PRIELIPP RC, BUTTERWORTH JF, ZALOGA GP et al. - Effects of amrinone on cardiac index, venous oxygen saturation, and venous admixture in patients recovering from cardiac surgery. Chest $1991 ; 99: 820-5$.

18.CARMONA MJC \& AULER JR JOC - Effects of inhaled nitric oxide on respiratory system mechanics, hemodynamics, and gas exchange after cardiac surgery. J Cardiothorac Vasc Anesth 1998; 12:157-61.

19.JOURNOIS D, PUARD P, MAURIAT P et al. - Inhaled nitric oxide as a therapy for pulmonary hypertension after operations for congenital heart defects. J Thorac Cardiovasc Surg 1994; 107:1129-35.

20. AZEKA E, AULER JR JOC, KAJITA L et al. Effects of low doses of inhaled nitric oxide combined with oxygen. Pediatric Cardiol 2002; 23(1)20-6.

Received for publication on May 18, 2001. 